\title{
Ss \\ Design of general apochromatic drift-quadrupole beam lines
}

\author{
C. A. Lindstrøm* and E. Adli \\ Department of Physics, University of Oslo, 0316 Oslo, Norway
}

(Received 24 March 2016; published 15 July 2016)

\begin{abstract}
Chromatic errors are normally corrected using sextupoles in regions of large dispersion. In low emittance linear accelerators, use of sextupoles can be challenging. Apochromatic focusing is a lesser-known alternative approach, whereby chromatic errors of Twiss parameters are corrected without the use of sextupoles, and has consequently been subject to renewed interest in advanced linear accelerator research. Proof of principle designs were first established by Montague and Ruggiero and developed more recently by Balandin et al. We describe a general method for designing drift-quadrupole beam lines of arbitrary order in apochromatic correction, including analytic expressions for emittance growth and other merit functions. Worked examples are shown for plasma wakefield accelerator staging optics and for a simple final focus system.
\end{abstract}

DOI: 10.1103/PhysRevAccelBeams.19.071002

\section{INTRODUCTION}

Chromatic errors are inherent to charged particle beam optics, due to the energy dependent kick exerted by a magnetic quadrupole, as seen explicitly in the normalized quadrupole strength

$$
k=\frac{e g}{p_{0}(1+\delta)},
$$

where $g$ is the quadrupole field gradient, $e$ is the particle charge, $p_{0}$ is the nominal particle momentum, and $\delta$ is the relative momentum offset. Coupled with an energy spread, this results in mostly unwanted nonlinear distortions of the beam.

Correcting chromatic errors is conventionally done using sextupoles in regions of large dispersion [1]. This method introduces nonlinear force terms, of which some may be canceled by careful lattice design, and adds additional dispersion and synchrotron radiation in the case of linear accelerators. In particular, this results in unfavorable energy vs length scaling laws for future high-energy, low emittance advanced accelerator concepts [2].

Fortunately, another method known as apochromatic focusing can be used to correct chromatic effects in linear accelerators without sextupoles or dipoles. Inspired by light ray optics, the aim is to simultaneously focus a range of colors (energies) to the same focal point using lenses (quadrupoles) only. Figure 1 illustrates this mechanism for both light beams and charged particle beams. In (a) three distinct colors are focused, while in (b) the energy dependence of the focusing is canceled to first order.

*c.a.lindstrom@fys.uio.no

Published by the American Physical Society under the terms of the Creative Commons Attribution 3.0 License. Further distribution of this work must maintain attribution to the author(s) and the published article's title, journal citation, and DOI.
This method was introduced in 1987 by Montague and Ruggiero [3] in an attempt to meet the requirements of the Compact Linear Collider (CLIC) [4] final focus system. Presenting an analytical solution for a thin-lens first-order

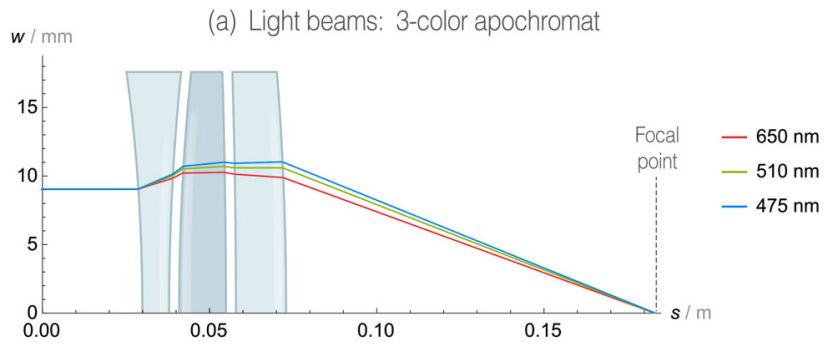

(b) Charged particle beams: first-order apochromat

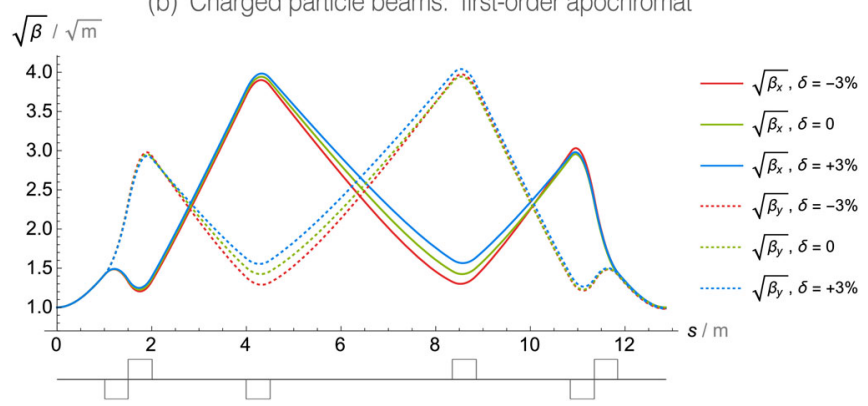

FIG. 1. Plot (a) shows width $w$ of three light beams vs the optical axis $s$ for a 3-color apochromat. Plot (b) shows transverse beam size $\sqrt{\beta}$ in $x$ and $y$ vs $s$ for a first-order apochromat. Both apochromats use the same principle: beams of different color/ energy are focused differently through the system, but end up focused to the same point. However, the two examples are different in a subtle way: In plot (a) three distinct colors are focused to the same point, leaving intermediate colors slightly unfocused. This is how achromatic lenses for light optics are often designed. In contrast, in plot (b) the nominal energy is perfectly focused, and the focusing error is canceled to first order in $\delta$, leaving small-offset energies well focused. In this paper, we study arbitrary order apochromats for charged particle beams. 
apochromatic telescope of arbitrary magnification, they concluded that while not providing sufficient energy acceptance for CLIC, the method was of considerable interest in less extreme cases. However, their contribution has until recently remained largely ignored.

Now, a surge in advanced accelerator research has lead to a renaissance of interest in apochromatic focusing. Advances on the topic were made by Balandin et al. in work motivated by requirements at the European XFEL [5], showing e.g. that any drift-quadrupole beam line has a set of unique first-order apochromatic Twiss parameters [6], as well as finding a proof-of-principle 20-quad first-order apochromatic FODO-lattice [7]. Looking ahead, challenging demands for tightly focused beams, high emittance preservation and short beam lines in emerging accelerator technologies such as laser- and plasma wakefield accelerators highlight the need for apochromatic beam line design.

Although earlier work has successfully demonstrated the plausibility of apochromatic focusing, mostly by analytical means and by employing various symmetries, it has not been sufficiently illustrated how to systematically construct such lattices in general. In this paper, we take a more general approach and present a methodical framework for computing apochromatic beam lines corrected to arbitrary order, both with and without the use of symmetric lattices.

\section{CHROMATICITY DEFINITIONS}

Before delving into how chromatic errors are canceled, it is necessary to distinguish between two closely related, but different quantities. The chromaticity

$$
\xi=\frac{1}{2 \pi} \frac{\partial \mu}{\partial \delta}
$$

where $\mu$ is the betatron phase advance [8], quantifies the chromatic error of a single particle, whereas the chromatic amplitude [9] or W-function

$$
W=\sqrt{\left(\frac{\partial \alpha}{\partial \delta}-\frac{\alpha}{\beta} \frac{\partial \beta}{\partial \delta}\right)^{2}+\left(\frac{1}{\beta} \frac{\partial \beta}{\partial \delta}\right)^{2}}
$$

quantifies the chromatic error of the beam as a distribution. In Eq. (3) we have used the definitions

$$
\begin{gathered}
\beta=\frac{\left\langle x^{2}\right\rangle}{\epsilon} \\
\alpha=-\frac{\left\langle x x^{\prime}\right\rangle}{\epsilon}=-\frac{1}{2} \frac{\partial \beta}{\partial s},
\end{gathered}
$$

where $x, x^{\prime}, \epsilon$ and $s$ are transverse position, angle, geometric emittance and longitudinal position. $\beta$ and $\alpha$ are better known as the Courant-Snyder or Twiss parameters [8] used to describe beam focusing in an accelerator lattice, and all conclusions in this paper are subject to the approximations of this framework. Note that throughout this paper, we will use $\frac{\partial}{\partial \delta}$ as shorthand for $\left.\frac{\partial}{\partial \delta}\right|_{\delta=0}$, i.e. the chromatic derivative evaluated at $\delta=0$.

Circular accelerators demand strict control of tune to avoid resonances, hence the chromaticity $\xi$ must be

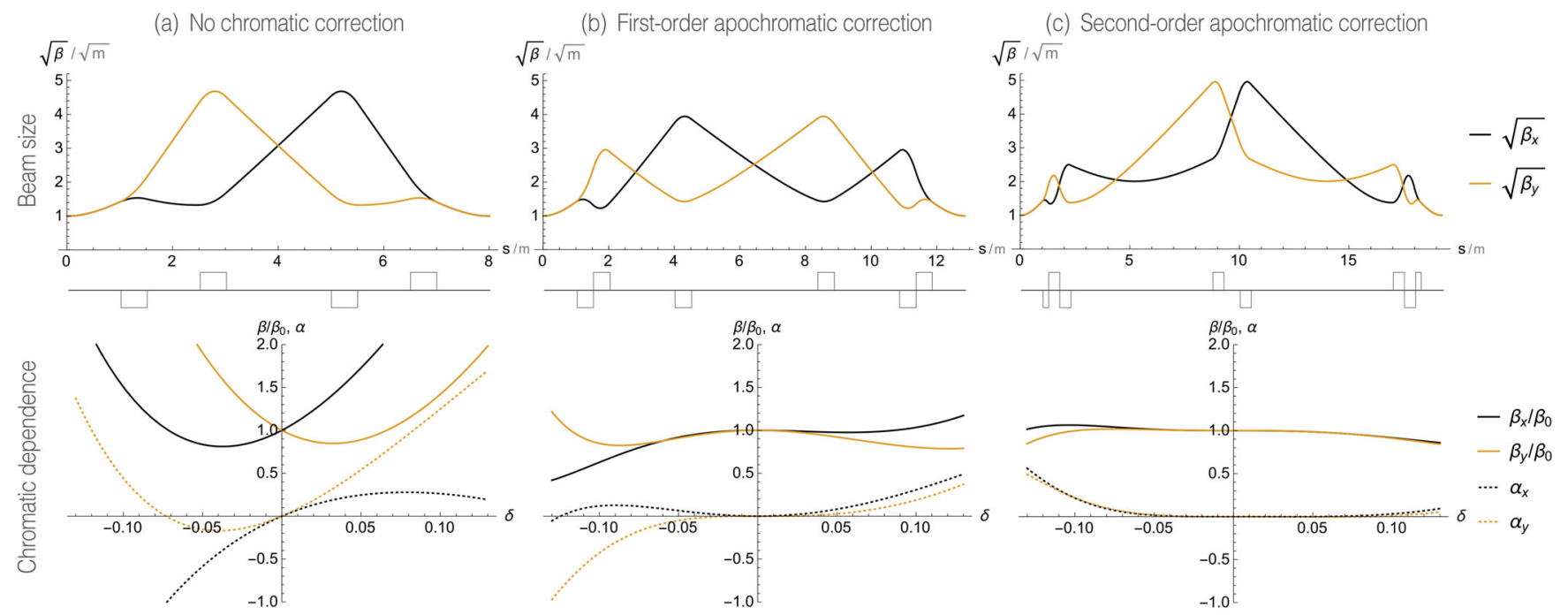

FIG. 2. Plots of $\sqrt{\beta}$ (proportional to rms beam size) vs beam line axis $s$, and chromatic dependence of $\beta(\delta)$ and $\alpha(\delta)$ vs $\delta$, shown for different orders of apochromatic focusing. All solutions satisfy initial and final Twiss parameters $\beta=1 \mathrm{~m}$ and $\alpha=0$ in both planes, with a $1 \mathrm{~m}$ drift before and after the first and last quadrupoles respectively. The chromatic dependence of the lattice flattens progressively with higher orders of apochromatic focusing; No chromatic correction (a) results in chromatic amplitude $W \neq 0$ (a slope) at nominal energy $\delta=0$, whereas first order correction (b) removes this chromatic amplitude $W=0$ (no slope), and second order correction (c) flattens it further by removing second order chromatic errors (curvature) around $\delta=0$. Overall, the chromatic dependence can be decreased at the cost of longer lattices with more quadrupoles, where the appropriate order of the correction is determined by the energy spread of the beam. 


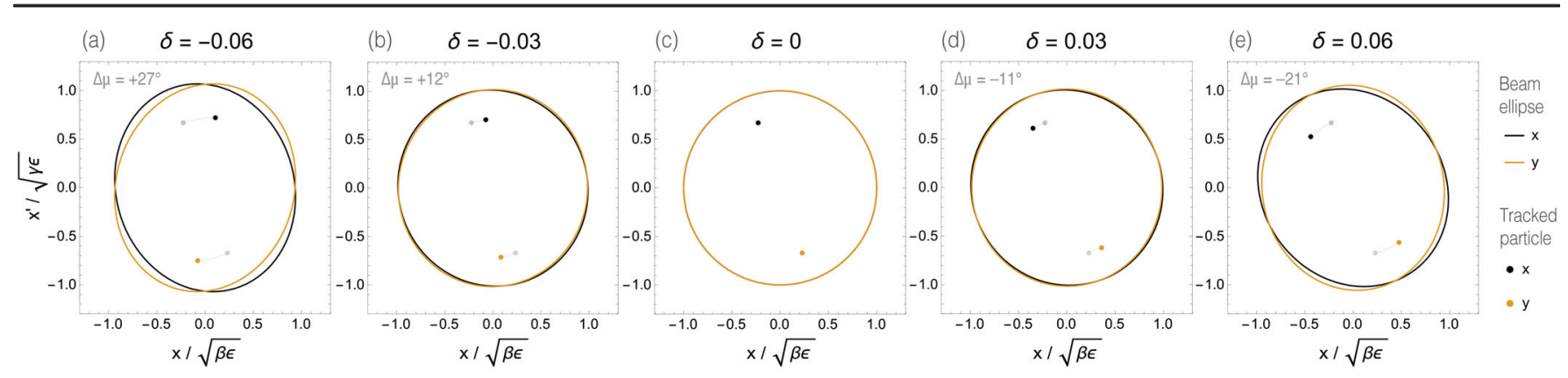

FIG. 3. Phase space plots with beam ellipses and a single tracked particle for several energy offsets $\delta$, after transport through the first order apochromatic lattice shown in Fig. 2(b). As the energy increases $[(\mathrm{a}) \rightarrow(\mathrm{e})]$, the particle experiences less phase advance $(\Delta \mu)$, indicating a nonzero chromaticity $\xi<0$ as defined by Eq. (2). However, since the distribution of particles retains the same shape around nominal energy [(b) and (d)], $\beta$ and $\alpha$ are unchanged to first order and therefore chromatic amplitude $W=0$. At larger energy offsets [(a) and (e)], the ellipse is distorted due to higher order errors.

canceled. This can only be done using nonlinear optics such as sextupoles [10]. However in single-pass linear accelerators, with no concept of tune and resonance, the chromaticity $\xi$ usually does not need to be canceled. On the other hand, to ensure the correct focusing, it is important to cancel the $W$-function, which indeed can be corrected using sextupole-free apochromatic focusing. In colliding-beam storage rings, a combination of both methods can be used, where the chromaticity is canceled using sextupoles in the arcs and the $W$-function is canceled using apochromatic focusing in the intersection regions.

Figure 2 illustrates in more detail how apochromatic lattices are used to reduce the chromatic dependence of beam focusing, while Fig. 3 elaborates on how the $W$-function (focusing energy dependence) can be canceled when chromaticity $\xi$ (phase advance energy dependence) is not.

\section{CHROMATIC EXPANSIONS}

In light ray optics, the standard approach to minimizing chromatic aberration for a given spectrum is to simultaneously focus a number of different colors [11]. This can also be done in beam optics. The underlying assumption is that by matching two narrowly offset energies, the energies between them are also approximately matched. To decrease the degree of mismatch in this region, more matched energies are added, requiring additional d.o.f.

In this paper, we employ a more systematic approach, namely to explicitly cancel terms in the chromatic expansion, defined as the Taylor series of a quantity with respect to relative energy offset $\delta$, e.g.,

$$
\begin{aligned}
\beta(\delta) & =\sum_{n=0}^{\infty} \frac{\delta^{n}}{n !} \frac{\partial^{n} \beta}{\partial \delta^{n}} \\
& =\beta_{0}+\frac{\partial \beta}{\partial \delta} \delta+\frac{1}{2} \frac{\partial^{2} \beta}{\partial \delta^{2}} \delta^{2}+\mathcal{O}\left(\delta^{3}\right) .
\end{aligned}
$$

A beam typically has a Gaussian or similarly distributed energy spread, where energies further from the nominal are progressively less populated. Minimizing the weighted average mismatch is accomplished most effectively by first canceling first order chromatic terms, then second order terms etc., adding only a constant number of additional d.o.f. per order. By directly taking control over the expansion Eq. (6), the lattice designer has more precise control of the chromatic performance of the beam line, as exemplified in the next section.

\section{EMITTANCE GROWTH IN DRIFT-QUADRUPOLE BEAM LINES}

Using drift-quadrupole beam lines allows us to make relatively simple, yet exact expressions for measuring performance; orders of magnitude faster to compute compared to particle tracking. This is because focusing fields are linear, which ensures that every energy slice of the beam preserves its rms emittance, or equivalently [8]

$$
\beta(\delta) \gamma(\delta)-\alpha(\delta)^{2}=1,
$$

where the Twiss parameter $\gamma=\left\langle x^{\prime 2}\right\rangle / \epsilon$ represents the divergence of the beam. As we are interested only in effects due to focusing and not dispersion (which a drift-quadrupole beam line does not introduce), we can ignore correlations between energy and phase space $\left(\langle x \delta\rangle=\left\langle x^{\prime} \delta\right\rangle=0\right)$, giving a squared projected emittance

$$
\begin{aligned}
\bar{\epsilon}^{2} & =\left\langle x^{2}\right\rangle\left\langle x^{2}\right\rangle-\left\langle x x^{\prime}\right\rangle^{2} \\
& =\left(\epsilon_{0} \bar{\beta}\right)\left(\epsilon_{0} \bar{\gamma}\right)-\left(\epsilon_{0} \bar{\alpha}\right)^{2} \\
& =\epsilon_{0}{ }^{2}\left(\bar{\beta} \bar{\gamma}-\bar{\alpha}^{2}\right),
\end{aligned}
$$

where $\epsilon_{0}$ is the geometric emittance of each energy slice, and a bar denotes energy average (projection). In a transport beam line, a natural merit function is the relative projected squared emittance growth 


$$
\frac{\Delta \epsilon^{2}}{\epsilon_{0}^{2}} \equiv \frac{\bar{\epsilon}^{2}-\epsilon_{0}^{2}}{\epsilon_{0}^{2}}=\bar{\beta} \bar{\gamma}-\bar{\alpha}^{2}-1
$$

Other applications, such as final focus systems or spectrometers, might use rms spot sizes $(\sim \sqrt{\bar{\beta}})$ as their merit function. We observe that in any drift-quadrupole lattice, chromatic correction of $\beta(\delta)$ and $\alpha(\delta)$ is sufficient, since $\gamma(\delta)$ is determined completely by Eq. (8).

Assuming a Gaussian energy distribution of rms energy spread $\sigma_{E}$ and substituting for chromatic expansions, we find

$$
\begin{aligned}
\bar{\beta} & =\int_{-\infty}^{\infty} \beta(\delta) \frac{e^{-\frac{\delta^{2}}{2 \sigma_{E}}}}{\sqrt{2 \pi} \sigma_{E}} d \delta \\
& =\int_{-\infty}^{\infty} \sum_{n=0}^{\infty} \frac{\delta^{n}}{n !} \frac{\partial^{n} \beta}{\partial \delta^{n}} \frac{e^{-\frac{\delta^{2}}{2 \sigma_{E}}}}{\sqrt{2 \pi} \sigma_{E}} d \delta \\
& =\sum_{n=0}^{\infty} \frac{1}{n !} \frac{\partial^{n} \beta}{\partial \delta^{n}} \frac{\sigma_{E}^{n}}{\sqrt{2 \pi}} \int_{-\infty}^{\infty} \lambda^{n} e^{-\frac{1}{2} \lambda^{2}} d \lambda,
\end{aligned}
$$

where the substitution $\lambda=\delta / \sigma_{E}$ is used. For odd offset orders the emittance growth at $+\delta$ and $-\delta$ are equal and opposite, canceling each other, such that only even orders $(n=2 m)$ contribute a nontrivial Gaussian integral

$$
\int_{-\infty}^{\infty} \lambda^{2 m} e^{-\frac{1}{2} \lambda^{2}} d \lambda=\sqrt{2 \pi}(2 m-1) ! !
$$

where !! is the double factorial: $n ! !=n(n-2)(n-4) \ldots$. Substituting Eq. (16) into (14) and simplifying factorials using $(2 m) ! !(2 m-1) ! !=(2 m) !$ and $(2 m) ! !=2^{m} m !$, we are left with

$$
\bar{\beta}=\sum_{m=0}^{\infty} \frac{\sigma_{E}^{2 m}}{2^{m} m !} \frac{\partial^{2 m} \beta}{\partial \delta^{2 m}}
$$

and similar expressions for $\bar{\alpha}$ and $\bar{\gamma}$ by simply substituting $\alpha$ or $\gamma$ in place of $\beta$. Expanding Eq. (12) in terms of energy spread $\sigma_{E}$ gives

$$
\frac{\Delta \epsilon^{2}}{\epsilon_{0}^{2}}=\sum_{m=1}^{\infty} \chi_{m} \sigma_{E}^{2 m}
$$

with coefficients

$$
\chi_{m}=\sum_{k=0}^{m} \frac{\left(\frac{\partial^{2 l} \beta}{\partial \delta^{2 l}} \frac{\partial^{2 k} \gamma}{\partial \delta^{2 k}}-\frac{\partial^{2 l} \alpha}{\partial \delta^{2 l}} \frac{\partial^{2 k} \alpha}{\partial \delta^{2 k}}\right)}{2^{m} l ! k !},
$$

where $l=(m-k)$. The expansion in Eq. (18) starts at $m=1$ because by Eq. (8) there is no constant term.
Somewhat misleadingly, Eq. (19) gives the impression that only even order chromatic derivatives contribute to emittance growth. However, different order chromatic derivatives of $\beta, \alpha$, and $\gamma$ are related by Eq. (8) and its derivatives

$$
\frac{\partial^{n}}{\partial \delta^{n}}\left(\beta \gamma-\alpha^{2}\right)=0
$$

where $n>0$. To illustrate, we expand Eq. (20) for $n=1$ and $n=2$, giving

$$
\begin{gathered}
\beta \frac{\partial \gamma}{\partial \delta}=2 \alpha \frac{\partial \alpha}{\partial \delta}-\frac{\partial \beta}{\partial \delta} \gamma \\
\frac{\partial^{2} \beta}{\partial \delta^{2}} \gamma+\beta \frac{\partial^{2} \gamma}{\partial \delta^{2}}-2 \alpha \frac{\partial^{2} \alpha}{\partial \delta^{2}}=2\left(\frac{\partial \alpha}{\partial \delta}\right)^{2}-2 \frac{\partial \beta}{\partial \delta} \frac{\partial \gamma}{\partial \delta}
\end{gathered}
$$

and simplify the lowest order emittance growth coefficient $\chi_{1}$ by first using Eq. (21), then Eq. (22), rearranging and finally using Eq. (3):

$$
\begin{aligned}
\chi_{1} & =\frac{1}{2}\left(\frac{\partial^{2} \beta}{\partial \delta^{2}} \gamma+\beta \frac{\partial^{2} \gamma}{\partial \delta^{2}}-2 \alpha \frac{\partial^{2} \alpha}{\partial \delta^{2}}\right) \\
& =\left(\frac{\partial \alpha}{\partial \delta}\right)^{2}-\frac{\partial \beta}{\partial \delta} \frac{\partial \gamma}{\partial \delta} \\
& =\left(\frac{\partial \alpha}{\partial \delta}\right)^{2}+\frac{\partial \beta}{\partial \delta} \frac{1}{\beta}\left(\frac{\partial \beta}{\partial \delta} \frac{1+\alpha^{2}}{\beta}-2 \alpha \frac{\partial \alpha}{\partial \delta}\right) \\
& =\left(\frac{\partial \alpha}{\partial \delta}-\frac{\alpha}{\beta} \frac{\partial \beta}{\partial \delta}\right)^{2}+\left(\frac{1}{\beta} \frac{\partial \beta}{\partial \delta}\right)^{2} \\
& =W^{2} .
\end{aligned}
$$

Substituting this into Eq. (18) gives an expression for the lowest order relative squared projected emittance growth

$$
\frac{\Delta \epsilon^{2}}{\epsilon_{0}^{2}}=W^{2} \sigma_{E}^{2}+\mathcal{O}\left(\sigma_{E}^{4}\right)
$$

or equivalently [using Eq. (12)] the relative rms projected emittance growth

$$
\begin{aligned}
\frac{\Delta \epsilon}{\epsilon_{0}} & =\frac{\bar{\epsilon}-\epsilon_{0}}{\epsilon_{0}}=\sqrt{1+\frac{\Delta \epsilon^{2}}{\epsilon_{0}{ }^{2}}-1} \\
& =\frac{1}{2} W^{2} \sigma_{E}{ }^{2}+\mathcal{O}\left(\sigma_{E}{ }^{4}\right) .
\end{aligned}
$$

This result shows that, to lowest order, relative projected emittance growth can be written simply in terms of energy spread and the $W$-function, implying that in order to cancel emittance growth to third order in energy spread, it is 
sufficient to cancel first order chromatic derivatives. Balandin et al. defines such a lattice as a third-order apochromat [7], referring to emittance growth, whereas we will consistently call it a first-order apochromat, referring to the canceled chromatic derivatives.

For first-order correction, two d.o.f. are required per plane, one for each of the first order chromatic Twiss ( $\alpha$ and $\beta$ ) derivatives, in addition to those needed for standard zeroth order matching. More generally, at order $2 n$ in emittance growth, Eq. (20) can substituted into Eq. (19) to eliminate $\gamma$ and all $2 n$th chromatic Twiss derivatives, leaving derivatives of order $(2 n-1)$ or lower. However, if also the lowest $(n-1)$ chromatic Twiss derivatives are canceled, only terms with zeroth and $n$th derivatives remain. We conclude that to cancel emittance growth to order $\mathcal{O}\left(\sigma_{E}{ }^{2 n+1}\right)$ in energy spread, we must cancel the first $n$ chromatic Twiss derivatives in both planes; in total $4 n$ d.o.f. Note the linear complexity $\mathcal{O}(n)$ of d.o.f. for apochromatic correction of order $n$.

\section{METHOD}

Our goal is to find an apochromatic beam line which satisfies appropriately chosen constraints. Previous work has found such solutions analytically in simple cases [3] or using symmetry [7,12]. To find solutions to a general nonsymmetric set of constraints, it is useful to take a computational approach. In particular, this requires calculation of chromatic derivatives, which can be accomplished in a number of ways. Given the simplistic equations describing apochromatic focusing, we have developed an analytic-numerical algorithm to find such beam lines: (1) Consider a beam line defined by a set of alternating drift spaces of lengths $\{d\} \in \mathbb{R}^{+}$, and quadrupoles of strengths $\{k\} \in \mathbb{R}$ and lengths $\{l\} \in \mathbb{R}^{+}$, of which some are left as variables $\{q\}$. (2) Insert a relative energy offset $\delta$ in the quadrupole strengths [see Eq. (1)]: $\{k\} \rightarrow\{k /(1+\delta)\}$. (3) Compute analytically the transfer matrix $\boldsymbol{R}(\{q\}, \delta)$ of the overall beam line. This will be a complicated nonlinear function of $\delta$. (4) Expand $\boldsymbol{R}$ as a series to the required order in $\delta$ about the nominal energy, $\delta=0$. (5) Express the output Twiss parameters $\alpha$ and $\beta$ in terms of $\boldsymbol{R}$ and input Twiss parameters $\alpha_{0}, \beta_{0}, \gamma_{0}$ using

$$
\left[\begin{array}{l}
\beta \\
\alpha \\
\gamma
\end{array}\right]=\left[\begin{array}{ccc}
C^{2} & -2 C S & S^{2} \\
-C C^{\prime} & C S^{\prime}+S C^{\prime} & -S S^{\prime} \\
C^{\prime 2} & -2 C^{\prime} S^{\prime} & S^{\prime 2}
\end{array}\right]\left[\begin{array}{l}
\beta_{0} \\
\alpha_{0} \\
\gamma_{0}
\end{array}\right]
$$

where $\left(C, S, C^{\prime}, S^{\prime}\right)=\left(R_{11}, R_{12}, R_{21}, R_{22}\right) \quad$ in $\quad x \quad$ and $\left(C, S, C^{\prime}, S^{\prime}\right)=\left(R_{33}, R_{34}, R_{43}, R_{44}\right)$ in $y$ [8]. (6) Truncate the $\alpha(\delta)$ and $\beta(\delta)$-series at the required order in $\delta$. (7) Extract expressions for chromatic derivatives of $\alpha$ and $\beta$ as given by Eq. (6) to form a set of constraints and/ or a merit function. (8) Solve the constraints and/or minimize the merit function using the d.o.f. $\{q\}$ available.
Apart from particularly simple cases, this must be solved numerically. (9) (Optional) Simplify the numerical solving by employing symmetries to reduce the number of constraints and d.o.f., including periodicity, mirror symmetry, and mirror antisymmetry (i.e., quadrupole polarities are switched).

This method can be applied both to thick and thin quadrupoles, although thin quadrupoles result in a great reduction in calculation time.

A simple, yet powerful code was developed based on this method, using a computer algebra package (Mathematica) for expressing constraints analytically and a numerical analysis package (MATLAB) for solving or minimizing them. This code was used to compute all solutions in Sec. VI, as well as Figs. 1(b), 2, and 3. Execution times using a standard laptop computer typically ranged from seconds to minutes.

It should be noted that first order apochromatic matching following this method can be achieved in existing accelerator design codes, including MAD [13], by matching chromatic amplitude $W$ and phase $\Phi$.

\section{APPLICATIONS}

In order to demonstrate this method, two practical applications of apochromatic beam lines are considered: (i) Staging optics for a plasma wakefield accelerator and (ii) a simple final focus system. Together, these examples show varying degrees of symmetry (high and low, respectively), demagnifications (none and large, respectively), and different merit functions (emittance growth and spot size, respectively). However they both share requirements for localized small beam sizes with comparatively long adjacent drift spaces $\left(L^{*}\right)$, hence large chromatic errors, and a strong incentive to minimize the overall beam line length. To illustrate higher order apochromats, two solutions are presented for the plasma wakefield accelerator: one firstorder apochromat using conventional quadrupoles, and a third-order apochromat using axially symmetric plasma lenses.

\section{A. PWFA staging optics}

Plasma wakefield acceleration [14,15] is one of several emerging advanced accelerator concepts, in which energy is transferred from a laser (LWFA) or particle (PWFA) drive beam to a trailing witness beam via a wakefield in the plasma. To achieve much higher energies than that of the drive beam, this process must be repeated in multiple stages; the two beams must be separated and the witness beam reinjected behind a fresh drive beam into the next plasma cell.

To avoid significant emittance growth in the plasma channel, the staging optics between cells must match the witness beam to a specific Twiss $\beta$. Assuming parameters used in a recent beam-driven PWFA linear collider study 


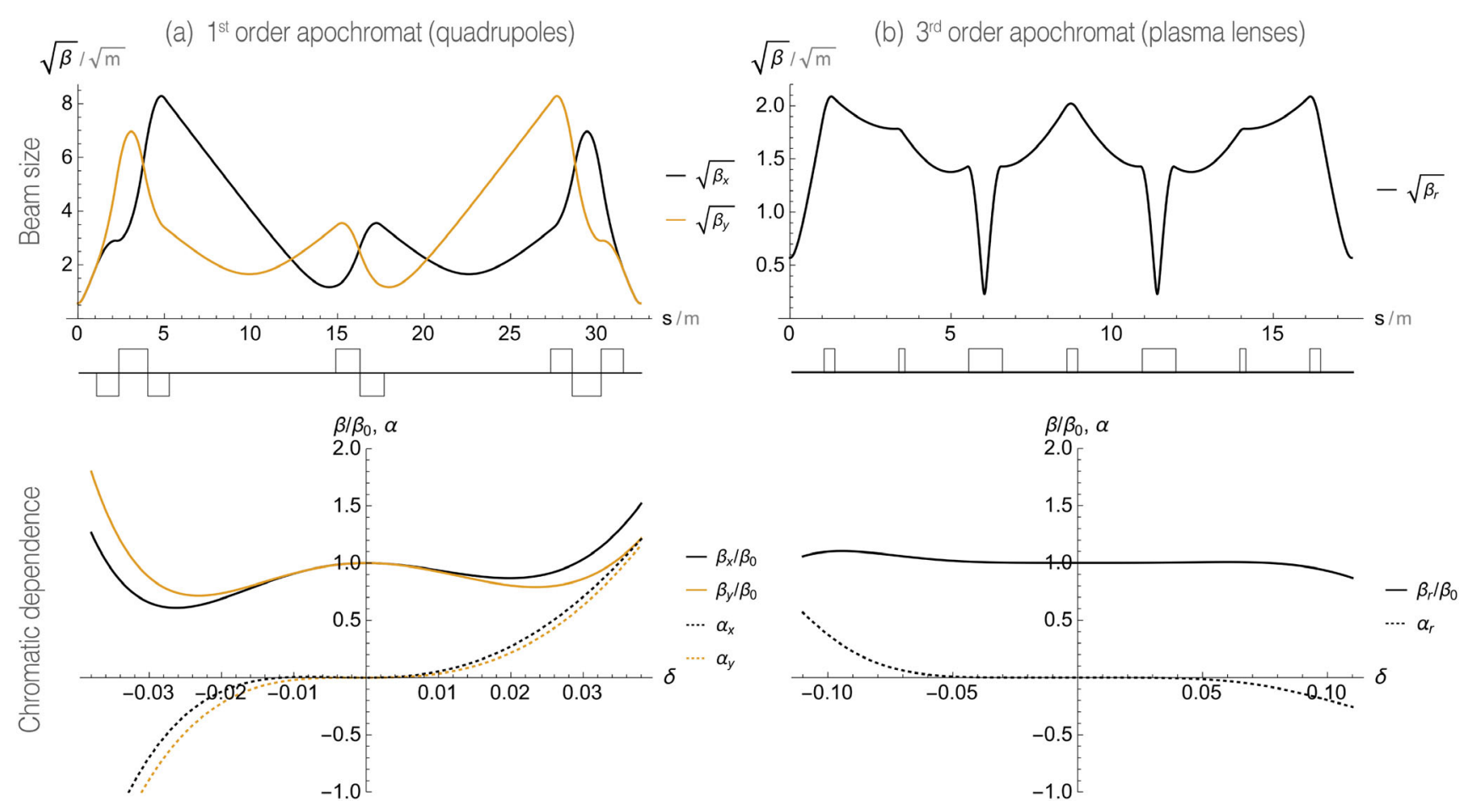

FIG. 4. Example A: PWFA staging optics, both using quadrupoles (a) and plasma lenses (b). Plots show $\sqrt{\beta}$ (proportional to rms beam size) vs beam line axis $s$, and chromatic dependence of $\alpha(\delta)$ and $\beta(\delta)$ vs offset $\delta$. Both solutions capture a $100 \mathrm{GeV}$ beam exiting a plasma (with density ramps) matched to $\beta_{0}=32.5 \mathrm{~cm}$ and refocuses it back to $32.5 \mathrm{~cm}$, with a $1 \mathrm{~m}$ drift space at the start and end for injection and extraction of drive beams. Solution (a) is a first-order apochromatic lattice using 8 quadrupoles with field gradient $160 \mathrm{~T} / \mathrm{m}$ are placed antisymmetrically (mirrored with polarity switched), whereas solution (b) is a third-order apochromatic lattice using 7 discharge capillary plasma lenses [18] with field gradient $3000 \mathrm{~T} / \mathrm{m}$ placed symmetrically. Transporting a beam with $1 \%$ rms energy spread leads to a projected emittance growth of $0.96 \%$ in lattice (a), and $0.000004 \%$ in lattice (b). Note the different $\delta$-scales in the two chromatic dependence plots.

[16], we use $\beta_{\text {mat }}=2.5 \mathrm{~cm}$ for a $100 \mathrm{GeV}$ witness beam, which has an energy spread of approximately $1 \%$. Using a plasma density ramp [17] of $\beta$-magnification 13, the staging optics must match to and from $\beta_{0}=32.5 \mathrm{~cm}$. Injection and extraction of drive beams introduce further considerations, discussed in Ref. [2]. Here we simply reserve $1 \mathrm{~m}$ of drift space at the beginning and end of the lattice for eventual injection and extraction sections.

The combination of large energy spread and tightly focused $\beta$-functions with long adjacent drift spaces results in significant chromatic errors that require cancellation. A naive beam line with no chromatic correction produces a projected emittance growth of around $10 \%$.

\section{First order quadrupole solution}

We start by solving the problem with lowest (first) order apochromatic correction using conventional magnetic quadrupoles. Since the problem is mirror symmetric, we can work with the first half of the lattice only, reducing the original 4 constraints $\left(\beta_{x}=\beta_{y}=\beta_{0}\right.$ and $\alpha_{x}=\alpha_{y}=0$ at the end) to only 2 constraints. Two solutions exist: mirror symmetry ( $\alpha_{x}=\alpha_{y}=0$ halfway) and mirror antisymmetry ( $\beta_{x}=\beta_{y}$ and $\alpha_{x}=-\alpha_{y}$ halfway), where quadrupole polarity is switched. We choose the latter for its similar emittance growth in both planes.

A first-order apochromat must satisfy both the zeroth order matching constraints and cancellation of their firstorder chromatic derivatives, resulting in a total of 4 constraints at the halfway point:

$$
\begin{gathered}
\beta_{x}-\beta_{y}=0 \\
\alpha_{x}+\alpha_{y}=0 \\
\frac{\partial \beta_{x}}{\partial \delta}-\frac{\partial \beta_{y}}{\partial \delta}=0 \\
\frac{\partial \alpha_{x}}{\partial \delta}+\frac{\partial \alpha_{y}}{\partial \delta}=0 .
\end{gathered}
$$

Since this requires 4 d.o.f., we define a half-lattice of 4 quadrupoles. To minimize the total length, we use quadrupoles of alternating maximum field strength, assumed to be $\pm 160 \mathrm{~T} / \mathrm{m}$, and vary their lengths. Solving Eqs. (32)-(35)) using the method outlined in Sec. V produces the solution presented in Fig. 4(a): a $32.5 \mathrm{~m}$ long lattice of 8 quadrupoles transporting a $100 \mathrm{GeV}$ beam of $1 \%$ energy spread 


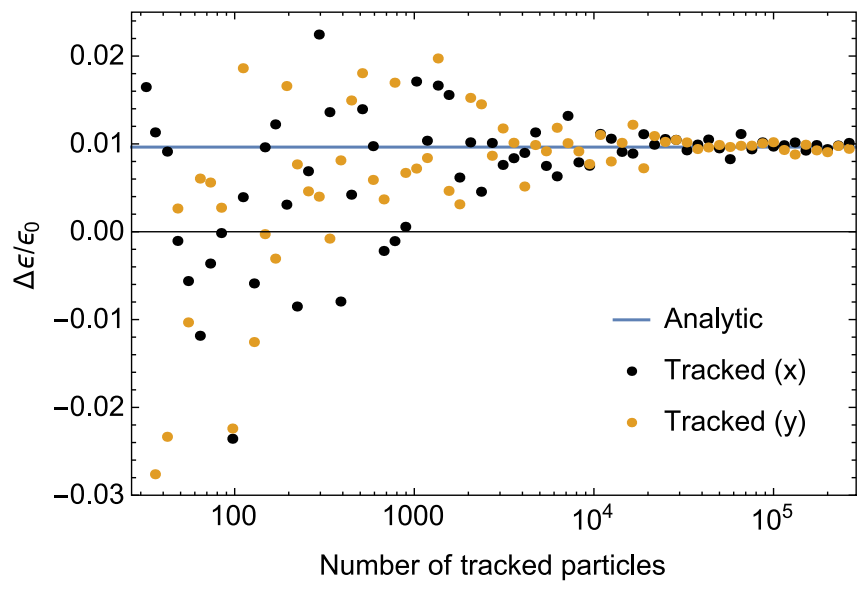

FIG. 5. Plot of relative projected emittance growth vs number of tracked particles, after Elegant-tracking [19] a beam with $1 \%$ rms energy spread through the apochromatic lattice shown in Fig. 4(a). Approaching large particle numbers, the tracked emittance growth converges to the analytic estimate given by Eq. (18), with an expected statistical error $(\sim 1 / \sqrt{N})$. Since the lattice is antisymmetric, the emittance growth is (very nearly) identical in both planes.

with a projected emittance growth of $0.96 \%$, which is around the limit for a linear collider application.

To illustrate the accuracy of the analytic expression for emittance growth Eq. (18), it is compared to the emittance growth of particles tracked through this example using Elegant [19], shown in Fig. 5. It verifies that as the number of tracked particles increase, the tracked emittance growth indeed converges to the analytically calculated value, which is many orders of magnitude faster to compute.

\section{Third-order plasma lens solution}

Emittance preservation can be dramatically improved with the use of axially symmetric lenses since the number of constraints is halved, which allows correction to higher order at low computational cost. An implementation of axially symmetric plasma lenses, based on strong discharge capillaries, has recently been presented by BELLA [18]. Constructing a third-order apochromat, using mirror symmetry, requires 4 constraints at the halfway point:

$$
\alpha_{r}=\frac{\partial \alpha_{r}}{\partial \delta}=\frac{\partial^{2} \alpha_{r}}{\partial \delta^{2}}=\frac{\partial^{3} \alpha_{r}}{\partial \delta^{3}}=0 .
$$

We define a half-lattice of 4 focusing-only plasma lenses of variable lengths, operating at $3000 \mathrm{~T} / \mathrm{m}$ [18]. Solving Eqs. (36) using the method in Sec. V, we obtain the solution shown in Fig. 4(b): a $17.4 \mathrm{~m}$ long lattice of 7 plasma lenses transporting a $100 \mathrm{GeV}$ beam of $1 \%$ energy spread with a projected emittance growth of only $0.000004 \%$, which is far below the requirements for a linear collider. In fact, the solution is almost completely achromatic up to an energy offset of $\pm 5 \%$.
A similarly performing solution of approximately half the length $(8.9 \mathrm{~m})$ can be found by using both focusing and defocusing lenses, as is possible in discharge capillary plasma lenses. However, the above example is used to show that apochromatic focusing can be used also in solenoids and plasma lenses where only focusing fields are available.

\section{B. Final focus system}

Apochromatic beam lines can also be used to magnify or demagnify charged particle beams, as shown analytically in Ref. [3], in which case the beam line will be asymmetric unlike in the previous example. A first-order apochromatic telescope can be constructed by matching $R_{12}=R_{21}=$ $R_{34}=R_{43}=0$ and canceling their chromatic derivatives $T_{126}=T_{216}=T_{346}=T_{436}=0$, using the method in Sec. V. However, for this example, we will consider a simple final focus system, using a similar, but not identical, criterion of a locally minimized average spot size for a given energy spread. This is useful in applications like colliders and fixed target experiments.

With no symmetry to reduce the number of constraints, we must simultaneously match to

$$
\begin{gathered}
\beta_{x}=\beta_{x}^{*} \\
\beta_{y}=\beta_{y}^{*} \\
\alpha_{x}=\alpha_{y}=0
\end{gathered}
$$

at the interaction point (IP). This requires 4 d.o.f., and any additional d.o.f. will be used to maximize the appropriate merit function: relative luminosity,

$$
\frac{\mathcal{L}}{\mathcal{L}_{0}}=\sqrt{\frac{\beta_{x}^{*} \beta_{y}^{*}}{\bar{\beta}_{x} \bar{\beta}_{y}}},
$$

or equivalently minimizing $\bar{\beta}_{x} \bar{\beta}_{y}$, where a bar denotes momentum averaging. According to Eq. (17), this is to lowest order the same as minimizing

$$
\frac{\partial^{2} \beta_{x}}{\partial \delta^{2}}+\frac{\partial^{2} \beta_{y}}{\partial \delta^{2}} .
$$

In practice, however, the numerical search is helped by also adding first order apochromatic correction:

$$
\frac{\partial \beta_{x}}{\partial \delta}=\frac{\partial \beta_{y}}{\partial \delta}=\frac{\partial \alpha_{x}}{\partial \delta}=\frac{\partial \alpha_{x}}{\partial \delta}=0 .
$$

In total this amounts to 8 constraints requiring 8 d.o.f., and a merit function to be minimized by any additional variables.

Since our goal is to greatly demagnify the beam, our system has a large inherent scale difference: $\beta^{*} \ll \beta_{0}$. This complicates the numerical search, as parameters are 

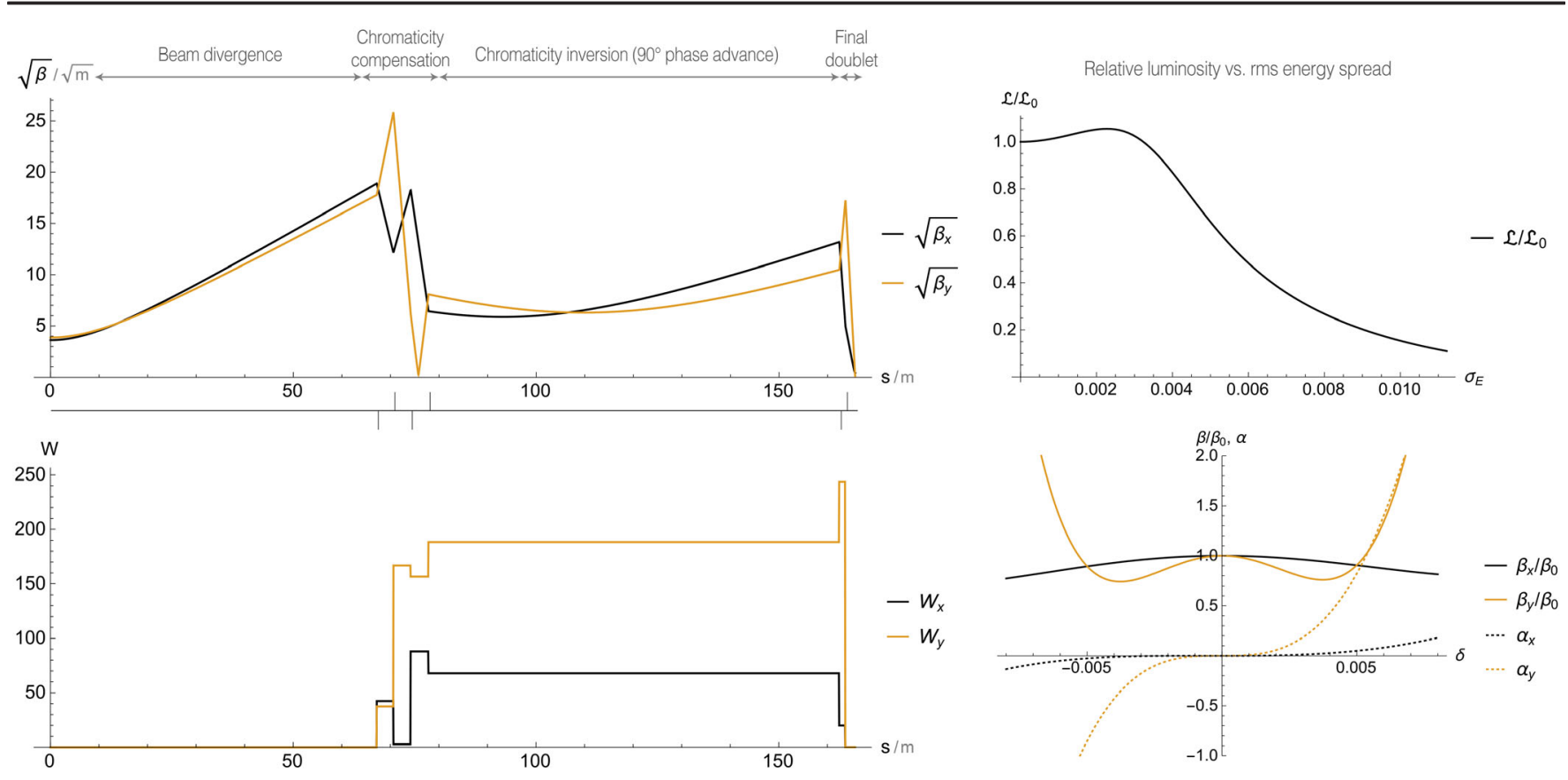

FIG. 6. Example B: Final focus. A $166 \mathrm{~m}$ long first-order apochromatic lattice using 6 (thin) quadrupoles, focuses a beam of $0.5 \%$ rms energy spread with about $30 \%$ luminosity loss. The beam is focused from $\beta_{x 0}=13.1 \mathrm{~m}, \beta_{y 0}=15 \mathrm{~m}$ down to $\beta_{y}^{*}=16.3 \mathrm{~cm}$, $\beta_{y}^{*}=1.33 \mathrm{~cm}$, in total a $\beta$-demagnification of 80 in $x$ and 1125 in $y$. The $W$-function is intentionally increased, then transported and inverted, until it is finally canceled in the final doublet, which focuses the beam to the interaction point.

simultaneously on large and small scales, resulting in a large fine-grained parameter space to be searched. Two methods are useful in mitigating this problem: (i) Backpropagate Twiss parameters for matching, starting with $\beta_{x}^{*}$, $\beta_{y}^{*}$ and matching to $\beta_{x 0}, \beta_{y 0}$. This greatly loosens matching tolerances. (ii) Find a solution for small demagnification (larger $\beta_{x}^{*}, \beta_{y}^{*}$ ), then use this solution as an initial guess for a slightly larger demagnification. Repeat this process until the desired demagnification is reached.

We start with Twiss parameters $\beta_{x 0}=13.1 \mathrm{~m}, \beta_{y 0}=15 \mathrm{~m}$, and $\alpha_{x 0}=\alpha_{y 0}=0$, focusing to $\beta_{x}^{*}=16.3 \mathrm{~cm}, \beta_{y}^{*}=1.33 \mathrm{~cm}$, using a lattice of 6 quadrupoles where the last drift space is $L^{*}=2 \mathrm{~m}$, we have 12 available d.o.f. (6 quadrupole strengths, 6 drift space lengths). Thin quadrupoles are used to speed up the numerical search. Applying the method in Sec. V, we obtain the solution shown in Fig. 6: a $166 \mathrm{~m}$ long lattice, focusing a beam of energy spread $0.5 \%$ by $\beta$-demagnifications 80 in $x$ and 1125 in $y$, with a luminosity loss of about $30 \%$ according to Eq. (40). Conceptually, this solution consists of four separate modules (see Fig. 6): the beam diverges to large $\beta$ 's; large chromatic amplitude $W$ is introduced; the chromatic amplitude is inverted by a $90^{\circ}$ phase advance; the beam is strongly focused and the firstorder chromatic amplitude is canceled. Note, however, that this structure was never imposed, but is simply a solution that results from applying the algorithm.

Ultimately, the performance of the presented solution compares unfavorably to state-of-the-art linear collider final focus systems, consistent with the conclusion in
Ref. [3]. For instance, the design proposed by Seryi and Raimondi [20] offers orders of magnitude larger demagnification at the same energy acceptance by employing local chromaticity correction, whereby sextupoles compensate chromatic errors as close to the source as possible, unlike our example which uses global chromaticity correction, by transporting artificially induced chromatic errors for some distance until it is finally canceled. Nevertheless, many applications require more moderate demagnification, in which case there can be significant benefits to using linear quadrupole optics, which does not distort the phase space as much as nonlinear sextupole optics.

An apochromatic final focus may, however, prove beneficial in future high energy circular electron-positron colliders, such as CEPC [21] or FCC-ee [22]. A major problem for these machines is hard X-rays hitting the particle collision detectors, produced in dipoles close to the IP as a nonzero dispersion is required for local chromaticity correction. By removing dipoles entirely from the IP area, an apochromat constitutes an interesting alternative approach to this problem, although only a careful study of all constraints can determine whether it provides a net improvement.

\section{CONCLUSION}

Apochromatic correction of chromatic focusing errors, which makes use of linear optics (quadrupoles) only, can be applied to preserve projected emittance of charged particle beams of significant energy spreads, in cases where tune 
resonances do not occur. This is especially relevant in emerging accelerator technologies like plasma wakefield acceleration. The method presented has been shown through examples to produce drift-quadrupole beam lines of any order in apochromatic correction, using both thick and thin quadrupoles, as well as varying degrees of symmetry. In addition, simple analytic expressions for emittance growth reduce the need for particle tracking to measure performance. We believe that apochromatic correction could be part of any advanced accelerator lattice designer's toolbox in the future.

\section{ACKNOWLEDGMENTS}

We thank A. Chao, Y. Nosochkov, R. Tomas, D. Schulte and J. Pfingstner for useful discussions. This work is supported by the Research Council of Norway.

[1] H. Zyngier, LAL Report No. 77/35, 1977.

[2] C. A. Lindstrøm, E. Adli, J. M. Allen, J. P. Delahaye, M. J. Hogan, C. Joshi, P. Muggli, T. O. Raubenheimer, and V. Yakimenko, Staging optics considerations for a plasma wakefield acceleration linear collider, Nucl. Instrum. Methods Phys. Res., Sect. A 829, 224 (2016).

[3] B. W. Montague and F. Ruggiero, CLIC Report No. Note 37, 1987.

[4] CLIC Conceptual Design Report, http://project-clic-cdr .web.cern.ch/project-clic-cdr/CDR_Volume1.pdf, 2012.

[5] V. Balandin, R. Brinkmann, W. Decking, and N. Golubeva, TESLA-FEL Report No. 2007-05, 2007.

[6] V. Balandin, R. Brinkmann, W. Decking, and N. Golubeva, Apochromatic beam transport in drift-quadrupole systems, arXiv:1305.1549.

[7] V. Balandin, R. Brinkmann, W. Decking, and N. Golubeva, Third-order apochromatic drift-quadrupole beamline, in Proceedings of the 3rd International Particle Accelerator Conference, New Orleans, LA, 2012 (IEEE, Piscataway, NJ, 2012), p. 1329.
[8] E. D. Courant and H. S. Snyder, Theory of the alternatinggradient synchrotron, Ann. Phys. (N.Y.) 3, 1 (1958).

[9] B. W. Montague, CERN LEP Report No. Note 165, 1979.

[10] A. Wolski, Beam Dynamics in High Energy Particle Accelerators, (Imperial College Press, London, 2014), p. 277.

[11] M. J. Herzberger and N. R. McClure, The design of superachromatic lenses, Appl. Opt. 2, 553 (1963).

[12] V. Balandin, R. Brinkmann, W. Decking, and N. Golubeva, Apochromatic Twiss parameters of drift-quadrupole systems with symmetries, in Proceedings of the 2nd International Particle Accelerator Conference, San Sebastián, Spain, 2011 (EPS-AG, Spain, 2011), p. 2163.

[13] F. C. Iselin, The MAD Program: Physical Methods Manual, 1994.

[14] R. Ruth, A. W. Chao, P. L. Morton, and P. B. Wilson, Report No. SLAC-PUB-3374, 1985.

[15] T. Tajima and J. M. Dawson, Laser Electron Accelerator, Phys. Rev. Lett. 43, 267 (1979).

[16] E. Adli, J. P. Delahaye, S. J. Gessner, M. J. Hogan, T. O. Raubenheimer, W. An, C. Joshi, and W. Mori, A beam driven plasma-wakefield linear collider: From Higgs factory to multi-TeV, arXiv:1308.1145.

[17] X. L. Xu et al., Exact phase space matching for staging plasma and traditional accelerator components using longitudinally tailored plasma profiles, arXiv:1411.4386.

[18] J. van Tilborg et al., Active Plasma Lensing for Relativistic Laser-Plasma-Accelerated Electron Beams, Phys. Rev. Lett. 115, 184802 (2015).

[19] M. Borland, User's Manual for Elegant, 2012.

[20] P. Raimondi and A. Seryi, Novel Final Focus Design for Future Linear Colliders, Phys. Rev. Lett. 86, 3779 (2001).

[21] F. Su et al., CEPC partial double ring lattice design, in Proceedings of the 7th International Particle Accelerator Conference, Busan, Korea, 2016 (JACoW, Geneva, 2016), p. 3785 .

[22] K. Oide et al., Design of beam optics for the FCC-ee collider ring, in Proceedings of the 7th International Particle Accelerator Conference, Busan, Korea, 2016 (JACoW, Geneva, 2016), p. 3821. 